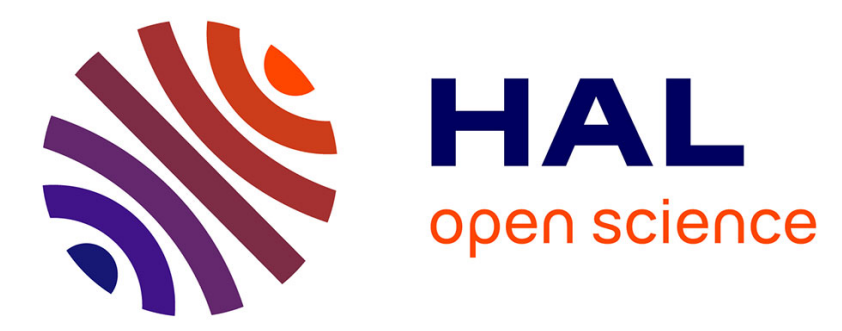

\title{
Karl Girardet illustrateur chez Mame, ou le voyage pittoresque à destination de la jeunesse
}

François Fièvre

\section{To cite this version:}

François Fièvre. Karl Girardet illustrateur chez Mame, ou le voyage pittoresque à destination de la jeunesse. Romantisme : la revue du dix-neuvième siècle, 2010, 147, pp.97-108. 10.3917/rom.147.0097 . hal-01164200

\section{HAL Id: hal-01164200 \\ https://hal.science/hal-01164200}

Submitted on 23 Jun 2015

HAL is a multi-disciplinary open access archive for the deposit and dissemination of scientific research documents, whether they are published or not. The documents may come from teaching and research institutions in France or abroad, or from public or private research centers.
L'archive ouverte pluridisciplinaire HAL, est destinée au dépôt et à la diffusion de documents scientifiques de niveau recherche, publiés ou non, émanant des établissements d'enseignement et de recherche français ou étrangers, des laboratoires publics ou privés.

\section{(ㅇ)(1) $\$$}

Distributed under a Creative Commons Attribution - NonCommercial - NoDerivatives| 4.0 


\section{KARL GIRARDET ILLUSTRATEUR CHEZ MAME, OU LE VOYAGE PITTORESQUE A DESTINATION DE LA JEUNESSE}

Par François Fièvre

Karl Girardet ${ }^{1}$ est peut-être, au XIX ${ }^{\mathrm{e}}$ siècle, l'artiste dont on retrouve le plus fréquemment la signature dans les ouvrages publiés par la maison tourangelle Mame, l'un des principaux éditeurs de livres catholiques pour la jeunesse du XIXe siècle, qui a poussé l'industrialisation du livre au maximum de ses potentialités. Pourtant son travail pour la maison Mame reste méconnu : tout d'abord parce que son œuvre, réputé mineur, est peu connu de manière générale; ensuite parce que les rares ouvrages s'attardant sur sa personnalité artistique se concentrent davantage sur sa carrière de peintre que d'illustrateur, et, au sein de sa carrière d'illustrateur, sur le chef-d'œuvre qu'est l'illustration de la Touraine (Mame, $1855^{2}$ ) au détriment de sa production pourtant très abondante à destination de la jeunesse.

Il ne s'agit pas, dans cet article, de prendre le contrepied de l'historiographie en tentant de montrer que les illustrations de Girardet pour la jeunesse seraient plus riches que son œuvre peint ou même que son illustration "pour adultes", mais de trouver le lien esthétique entre sa peinture et son œuvre illustré, entre son illustration pour la jeunesse et le chef-d'œuvre qu'est la Touraine. A travers l'exemple privilégié de la contribution de Girardet pour la maison Mame, nous voulons aussi mettre en valeur la manière dont la littérature pédagogique pour la jeunesse a su se réapproprier, à partir de la monarchie de Juillet, le modèle $d u$ voyage pittoresque romantique tel qu'il avait été lancé par le baron Taylor dans les années 1820.

Devant l'abondance de la production de l'artiste, nous laissons beaucoup de ses œuvres de côté et choisissons un angle d'analyse qui permette également de restituer une cohérence à sa carrière ${ }^{3}$ : cet angle est celui des liens qui unissent la peinture d'histoire et le paysage, et qui, dans ce genre intermédiaire qu'est le "voyage pittoresque », permet de donner à lire et à contempler l'histoire et la géographie aux jeunes lecteurs des livres de prix publiés par Mame.

\footnotetext{
1 Cet article a été rédigé dans le cadre d'un post-doctorat au sein du projet ANR « La maison Mame à Tours (1796-1975) : deux siècles d'édition pour la jeunesse ». Nous tenons à exprimer nos plus vifs remerciements à Cécile Boulaire pour son soutien constant lors de nos recherches. ${ }^{2}$ La Touraine de Bourassé, illustré par Français et Girardet, obtient lors de l'exposition universelle de Paris, en 1855, une grande médaille d'honneur pour l'éditeur, ainsi que onze récompenses pour les collaborateurs, dont une croix de chevalier de la Légion d'honneur pour le typographe Henri Fournier. L'ouvrage est désigné comme un « monument » bibliophilique par Gautier dans le Moniteur universel du 31 décembre 1856.

${ }^{3}$ Nous laisserons notamment de côté les illustrations pour les classiques (Fables de La Fontaine) et les œuvres de fiction (contes du chanoine Schmid, romans de Mme Woillez, Mes Prisons de Silvio Pellico, etc.).
} 


\section{Aperçu biographique}

Karl Girardet est un artiste d'origine suisse, mais dont la quasi intégralité de la carrière s'est déroulée en France, à Paris plus spécifiquement. Il est né au Locle, en Suisse, le 13 mai 1813, au sein d'une famille d'artistes et d'artisans active depuis déjà deux générations ${ }^{4}$. Son grand-père, Samuel, était libraire et imprimeur, son père, Charles-Samuel, peintre et graveur, et ses deux frères cadets, Édouard et Paul, deviennent eux-mêmes respectivement peintre et graveur. La famille de Charles-Samuel vit à Neuchâtel jusqu'en 1822, date à laquelle elle déménage à Paris. Des trois frères, Karl sera celui qui passera vraisemblablement le plus de temps dans la capitale française : Édouard vivra très longtemps à Brienz, en Suisse ; Paul reviendra s'installer plus tard, pour des raisons conjugales, à Neuchâtel ; Karl passera la fin de sa vie à Paris et mourra en 1871, de faim et de froid, dans la capitale assiégée durant la Commune.

Arrivé à Paris à l'âge de neuf ans, Karl Girardet envisage d'emblée, probablement par mimétisme familial, une carrière d'artiste. Il rentre dans l'atelier de Hersent, qui passe peu après sous la houlette de Léon Cogniet, peintre prometteur qui avait obtenu le premier Prix de Rome en 1817. Cogniet se fera durant sa carrière davantage remarquer pour son activité d'enseignant que pour ses propres peintures, quoiqu'au Salon de 1843 il obtienne un certain succès avec Le Tintoret peignant sa fille morte ${ }^{5}$. Toutefois, dans les années 1820 , Cogniet a une bonne réputation de peintre d'histoire et de genre historique, ce qui lui permet, au moment de la décoration du Musée historique de Versailles en 1837, d'obtenir quelques menues commandes qu'il exécute avec l'aide de certains de ses élèves : il signe notamment avec Karl Girardet la Bataille d'Héliopolis, scène de la campagne d'Égypte de Napoléon.

À partir de 1833, Karl Girardet passe trois années consécutives en Suisse, où il s'exerce à l'art du paysage. Le peintre affiche ainsi dans les années 1830 deux genres de prédilection : la peinture d'histoire ou de genre historique, du fait de l'enseignement de Léon Cogniet, et le paysage avec figures, par tropisme personnel et du fait de ses nombreux voyages. De 1839 à 1842, il peint pour la ville de Neuchâtel l'Assemblée de protestants surpris par les troupes catholiques (musée de Neuchâtel), qui connait un certain succès à Paris au Salon de 1842. C'est au tournant des années 1830 et 1840 que Girardet passe le plus de temps sur les routes, où il gagne sa réputation de peintre voyageur. Il part à Venise en 1838, à Rome en 1840, puis à Naples en 1842, enfin en Égypte à la fin de l'année $1842^{6}$. De son voyage en Égypte, il rapporte une abondante documentation iconographique, qu'il utilise en publiant des dessins orientalisants dans le Magasin pittoresque ${ }^{7}$. À cette époque Karl Girardet est quasiment un dessinateur

\footnotetext{
4 Trois ouvrages retracent l'histoire de cette famille d'artistes, dont le plus utile est paradoxalement le plus ancien : BACHELIN, Auguste, les Girardet, une famille d'artistes neuchatelois. Neuchâtel : Wolfrath et Metzner, 1870 (ci-après noté BACHELIN, 1870) ; BuRNAND, René, l'Étonnante Histoire des Girardet, artistes suisses, préface de Daniel Baud-Bovy. Neuchâtel : La Baconnière, 1940 (BuRnAND, 1940) ; BuRnAND, René, les Girardet au Locle et dans le monde. Neuchâtel : la Baconnière, 1957(BuRnAND, 1957).

${ }^{5}$ Léon Cogniet, 1794-1880 ; cat. expo. Orléans. Orléans : (s. n.), 1990.

6 BACHELIN, 1970, p. 64.

${ }^{7}$ Aurenche, Marie-Laure, (« Découvrir l’Égypte sans quitter Paris : l'itinéraire du Magasin pittoresque à deux sous (1833-1870) », in Romantisme. Paris, n²120, vol. 33, année 2003.
} 
attitré de l'hebdomadaire : 57 de ses œuvres sont répertoriées dans les tables décennales de 1842, ce qui en fait l'artiste qui a le plus collaboré durant cette décennie au Magasin pittoresque ${ }^{8}$. Sa participation ne se limite pas aux sujets égyptiens : il fournit également, avec son frère Paul, nombre de vues d'Italie et de Suisse, ainsi que des scènes de genre moralisantes.

Girardet semble être par ailleurs sinon le favori, du moins l'un des artistes que Louis-Philippe a comblé de ses commandes ${ }^{9}$. Le monarque le commissionne notamment pour immortaliser un déjeuner dans la forêt d'Eu avec la reine Victoria, récemment intronisée, ainsi que pour accompagner et peindre, en 1846, le duc de Montpensier lors de son mariage à Madrid avec l'infante d'Espagne. C'est également grâce à l'aide financière de Louis-Philippe que Karl et son frère Édouard peuvent partir, en 1842, à Alexandrie pour se documenter en vue de deux tableaux sur l'histoire des Croisades pour le Musée historique de Versailles ${ }^{10}$.

La fin de la monarchie de Juillet perturbe profondément la carrière et la vie de Girardet, non seulement parce qu'il était un peintre apprécié de LouisPhilippe, mais aussi parce qu'il a été choqué du sac du musée du Louvre pendant les émeutes. Il s'exile donc à Brienz pour une période qui reste toutefois indéterminée. Ses œuvres, en tout cas, reparaissent au Salon de Paris dès les années 1850. A l'Exposition universelle de Paris, en 1855, il expose une Vue de la cathédrale de Tours $^{11}$ ainsi qu'un paysage suisse et une scène de genre égyptienne : deux paysages ou "vues » contre une scène de genre, qui montrent bien le virage que l'artiste prend, à la fin de sa vie, vers le paysage. À partir des années 1860, en effet, d'après Auguste Bachelin ${ }^{12}$, le peintre se consacre presque entièrement au paysage, délaissant la scène de genre comme le genre historique : conséquence probablement d'une évolution du goût du public, mais sans doute aussi de la nécessité d'assumer une réputation de " peintre voyageur ».

À l'époque de ses débuts pour la maison Mame, en plus des commandes personnelles de Louis-Philippe, des œuvres exposées au Salon (paysages et scènes de genre) et des illustrations pour le Magasin pittoresque, il faut ajouter deux entreprises moins importantes quantitativement, mais qui restent remarquables : la participation, avec et sans Léon Cogniet, à la décoration du Musée historique de Versailles, grand musée consacré à l'histoire nationale réalisé sous Louis-Philippe ${ }^{13}$ ainsi que l'interprétation pour gravure sur bois, à partir de 1842, des dessins lithographiés de Rodolphe Töpffer pour les Voyages en zigzag (1844) et les Nouveaux voyages en zigzag (1854) ${ }^{14}$.

\footnotetext{
${ }^{8}$ Aurenche, Marie-Laure, Édouard Charton et l'invention du Magasin pittoresque (1833-1870). Paris : Honoré Champion, 2002, p. 475-479 et p. 283.

${ }^{9}$ BURNAND, 1940, p. 183 sq.

10 BACHELIN, 1870, p. 64-65.

11 Sujet à mettre en rapport avec la Touraine qui est aussi présenté à l'Exposition universelle de 1855 (voir supra, note 2)

12 Ibidem, p. 80.

13 Sur ce sujet, voir notamment GAEHTGENS, Thomas W., Versailles, de la résidence royale au musée historique, trad. Patrick Poirot. Paris : Albin Michel, 1984.

14 On remarquera que Mame assure en 1857 une nouvelle impression des Nouveaux voyages en zigzag (Archives départementales d'Indre-et-Loire, T848, déclaration d'intention d'imprimer du 4 juillet). Sur les différentes éditions des Voyages en Zigzag, voir KaEnEL, Philippe, « Les Voyages illustrés », dans Töpffer. Genève : Skira, 1996, p. 217 sqq.
} 


\section{À l'école de l'histoire : les scènes de genre historique}

Les premières illustrations signées par Girardet chez Mame semblent paraître en 1842 avec le Trésor littéraire des Jeunes Personnes, anthologie littéraire éditée par M. J. Duplessy et publiée dans la collection «Bibliothèque de la jeunesse chrétienne » (B.J.C.), collection qui avait été fondée par Mame à la fin de l'année 1836. Karl Girardet devient par la suite l'un des illustrateurs favoris de l'entrepreneur ${ }^{15}$. Si l'artiste a travaillé avant 1842 pour l'éditeur, il n'a pas signé de son nom complet, et c'est en tout cas après le lancement de la B.J.C. qu'il est fait appel à luii ${ }^{16}$.

En 1843, Girardet dessine quatre illustrations pour l'Histoire de Napoléon 1er d'Amédée Gabourd. Le frontispice représente Napoléon enfant, lisant Arrien, Polybe, Plutarque dans la cour d'école. Alors que les autres enfants jouent, Napoléon se fait donc déjà remarquer par ses professeurs, qui tournent la tête vers lui plutôt que vers la cohue qui fait frise en arrière-plan. L'exemple de l'instruction et de la lecture est d'emblée donné au jeune lecteur, qui s'identifie d'autant plus au futur empereur qu'est ménagée une mise en abyme de l'acte de lecture à l'endroit symbolique de l'ouverture du livre : de même que Napoléon lit la vie des empereurs romains dans Plutarque et d'Alexandre le Grand dans Arrien, les jeunes lecteurs des livres de Mame peuvent lire la vie de l'empereur Napoléon dans Amédée Gabourd. Une continuité est ainsi ménagée entre l'empire romain et le Premier Empire, qui témoigne peut-être des affinités de la famille Mame avec la cause impériale : ce sera en tout cas la famille politique d'Ernest quand il deviendra, de 1849 à 1865, maire de Tours.

La vignette de page de titre, de son côté, vient confirmer l'allégeance de Mame à Rome en montrant l'épisode de l'extrême onction de l'Empereur déchu, qui vient réinscrire l'histoire napoléonienne dans le giron de l'Église. Du frontispice à la vignette de titre, on passe donc non seulement de l'enfance de Napoléon à sa mort, mais également d'un message politique à un message religieux. Non seulement l'ouverture du livre forme un condensé de la vie de l'empereur, de sa première éducation à sa mort, mais elle permet également de dégager une évolution, des premières préoccupations temporelles, alimentées par la lecture des auteurs romains, aux aspirations spirituelles.

Les deux scènes, comme d'ailleurs les autres planches qui illustrent le livre de Gabourd, ne représentent jamais d'épisode historique fondamental de la vie de l'empereur français, mais plutôt des anecdotes jugées significatives ou pittoresques. Bonaparte exhortant ses troupes avant la campagne d'Italie permet de mettre en avant la figure du général proche de ses soldats, sans piédestal autre que celui d'un léger enrochement. Napoléon tenant une cour princière à Dresde ménage un lien cette fois-ci non plus avec les empereurs romains, mais avec les habitudes luxueuses de la monarchie française.

\footnotetext{
15 À travers un témoignage de Giacomelli, Émile Bayard rapporte, dans l'Illustration et les illustrateurs (Paris : Delagrave, 1898, p. 156-159), que Girardet était considéré par Alfred Mame comme «l'un de ses dessinateurs favoris ».

16 Une récente communication d'Olivia Voisin (janvier 2010, Tours, inédit) a mis en évidence une première intervention de Girardet chez Mame dès 1838, ainsi que le fait que l'artiste était, parmi les illustrateurs de la $2^{\mathrm{e}}$ série de la BJC, celui dont on retrouvait le travail le plus fréquemment entre 1836 et 1850.
} 
Ce caractère anecdotique permet d'inscrire les illustrations de Girardet dans le registre de la peinture de genre historique, qui voit le jour au tout début du $\mathrm{XIX}^{\mathrm{e}}$ siècle avec la peinture troubadour ${ }^{17}$, mais se poursuit sous d'autres formes bien avant dans le siècle. Ségolène Le Men rappelle que les genres, dans le livre illustré, diffèrent sensiblement de ceux en vigueur dans la peinture académique : là où la peinture de Salon voyait traditionnellement ses œuvres réparties en peinture d'histoire, portrait, paysage, scène de genre et nature morte, l'art de l'illustrateur qui se met en place durant la période romantique est plus fréquemment classé en scènes, vues, types et portraits ${ }^{18}$. Les scènes peuvent toutefois être classées d'une part en scènes historiques, d'autre part en scènes de genre, selon, justement, le genre de texte qui est illustré : histoire ou fiction. Dans les genres intermédiaires comme le roman historique, qui connait une vogue certaine durant la période romantique sur le modèle des œuvres de Walter Scott, la limite devient toutefois assez indéterminée, et les illustrations comme celles de Girardet pour le Napoléon de Gabourd ont moins pour but de célébrer un moment historique ou de représenter un épisode fictif de roman que de divertir en instruisant et vice-versa. L'image a une fonction divertissante du fait du rapport de proximité symbolique ménagé entre le moment représenté et la situation ou les préoccupations sociales du lecteur attendu: le lecteur du Napoléon de Gabourd ne demande pas seulement à être instruit des différents faits et gestes politiques du chef d'État, mais demande également à être projeté dans l'itinéraire rêvé et émouvant d'un petit lieutenant corse devenu empereur de la moitié de l'Europe, d'un général qui reste proche de ses soldats, et qui en même temps mène une vie princière, luxueuse, idéal des aspirations bourgeoises. L'œuvre de Girardet s'inscrit donc en partie dans la scène de genre historique, ou "genre anecdotique », qui caractérise l'exploitation des thèmes historiques dans l'art de l'époque ${ }^{19}$.

\section{Le peintre voyageur : les paysages}

En 1846, dans la vignette de titre du Voyage en France de Mme Amable Tastu $^{20}$, Girardet met en images un autre type de lecture que celle du livre d'histoire: celle d'une carte de géographie. La vignette représente un père montrant à son fils et à sa fille une carte, telle que celle qu'on retrouve à la fin de l'ouvrage même. Le voyage que propose en effet l'auteur dans son introduction est celui d'un père qui, occupé par son travail pendant les vacances de ses enfants, leur promet de les faire voyager de manière abstraite à travers ses

\footnotetext{
17 PuPIL, François, le Style troubadour, ou la nostalgie du bon vieux temps. Nancy : Presses universitaires de Nancy, 1985.

18 LE MEN, Ségolène, la Cathédrale illustrée de Hugo à Monet, regard romantique et modernité. Paris : CNRS Editions, 2002 (1998), p. 111 sq.

${ }^{19}$ Chaudonneret, Marie-Claude, « Du « genre anecdotique » au « genre historique », une autre peinture d'histoire », dans les Années romantiques, la peinture française de 1815 à 1850, Isabelle Julia et Jean Lacambre (dir.) ; cat. expo. Nantes, Paris, Plaisance. Paris : Réunion des Musées Nationaux, 1995, p. 76-85.

${ }^{20}$ Aucune indication ne précise que ce volume est destiné aux enfants : pas de préface ou d'appartenance à une collection particulière. Mais l'introduction met en scène deux enfants en discussion avec leur père, scène qui commence par l'évocation du repos du fils, qui à la fin de l'année scolaire est entouré de ses livres de prix. Ce qui, placé en incipit de l'ouvrage, constitue une mise en abyme évidente du livre que le lecteur tient entre ses mains.
} 
cartes, ses notes et ses propres souvenirs de voyage : «Tous les soirs, sans sortir de notre petit jardin [...], nous partirons de cette capitale pour quelqu'une de nos grandes villes.» (p.4) C'est cette scène d'énonciation, de récit-cadre, que Girardet représente en position liminaire. Ce faisant, il opère un contraste entre l'intimité de la scène, qui a lieu dans un jardin clos par une nuit d'été, et la rêverie géographique occasionnée par la lecture des cartes - car d'autres cartes semblent attendre qu'on les déroule dans le carton apposé au pied de la chaise du père. Réexploitant de manière didactique le thème fantaisiste du "voyage autour de ma chambre », cette petite vignette aux bords évanescents propose d'entrer dans une rêverie géographique qui est davantage celle du livre - et en particulier celle du livre illustré de vues - que celle du voyageur allant de luimême par monts et par vaux.

Le voyage des deux enfants débute par un itinéraire de Paris vers Lyon. Ils passent alors devant le château de Vincennes, et une gravure sur bois judicieusement placée au milieu de la phrase fait jaillir le donjon de Vincennes du blanc de la page, concrétisant le voyage pour ainsi dire virtuel des vacanciers : "Avant de passer le pont de Charenton, au confluent de la Seine et de la Marne, jetons un regard sur notre gauche, et disons adieu [gravure] au château de Vincennes et à son donjon, sombre masse quadrangulaire à cinq étages, flanquée de tourelles, qui domine si tristement toute la plaine. » (p.9) Pour rendre la plaine moins triste et plus attrayante que ne le suggère le texte, Girardet ajoute l'artifice pittoresque d'un arbre, qui revêt plusieurs fonctions. Il fournit un repère spatial par rapport auquel on comprend que le château de Vincennes se trouve au loin, mais pas au dernier plan cependant: un bâtiment plus sombre laisse deviner un troisième degré de profondeur, qui vient compléter la grille canonique du paysage pittoresque, avec un premier plan sombre et deux autres plans plus clairs, afin de bien clarifier l'espace, de le rendre «lisible» et parcourable ${ }^{21}$. L'arbre sert également, au premier plan, à distinguer l'espace du spectateur de celui de la représentation, faisant repoussoir autant que cadre à l'intérieur duquel se déploie l'image du monument. Enfin, c'est un accident qui vient égayer la « triste » plaine, il apporte la touche d'irrégularité qui caractérise l'esthétique pittoresque depuis son invention dans la Grande-Bretagne du XVIII ${ }^{\mathrm{e}}$ siècle ${ }^{22}$.

On retrouve la même esthétique pittoresque, faite à la fois de symétrie et d'irrégularité, dans la vignette qui agrémente la page 28. Les deux montagnes suisses, si connues de Girardet, se répondent de manière symétrique de manière à ne pas frapper l'œil par une impression trop forte qui caractériserait, à la différence du pittoresque, l'esthétique du sublime. L'illustrateur évite cependant la monotonie classique en arrangeant l'un des flancs de manière irrégulière, en faisant l'une des berges plus longue que l'autre dans l'espace de la

\footnotetext{
${ }^{21}$ ANDREWs, Malcolm, The Search for the Picturesque, Landscape Aesthetics and Tourism in Britain, 1760-1800. Aldershot: Scolar Press, 1989, 2e chapitre. À la différence du paysage classique néanmoins, où les fonds tirent vers le clair, l'arrière-plan est ici plus sombre que le plan intermédiaire. Conséquence peut-être du passage à la gravure sur bois, qui vient ici traduire l'indistinction des arrière-plans par une tonalité plus sombre (la traduction aurait sans doute été différente dans une gravure sur acier, qui permet, grâce à l'étagement des morsures de l'eauforte, davantage de nuances que le bois), mais sans doute aussi de l'adoption d'une « recette » de composition différente de celle léguée par les classiques.

22 Voir ibidem, et, de manière plus théorique, les écrits de William Gilpin.
} 
représentation, en cadrant de manière à inclure la ville dans le champ, enfin en ajoutant le détail, pittoresque s'il en est, des pêcheurs et du bateau à voile, dont le mat se situe dans l'axe vertical médian de la vignette, afin de ne pas trop dynamiser l'image. Les figures servent bien évidemment à donner l'échelle, ainsi qu'à fournir un référent humain auquel on puisse s'identifier quand on contemple l'image. L'impression donnée est alors celle d'une montagne sereine, idyllique, tranquille, qui correspond davantage à l'imaginaire de la Nouvelle Héloïse qu'à celui des terribles abîmes peints par Turner ou de Loutherbourg 23 .

Dans un genre plus exotique, Girardet exploite sa connaissance des paysages égyptiens quand il met en images Un hiver en Égypte d'Eugène Poitou (1860). L'ouvrage ne semble pas spécialement destiné aux enfants, mais ses gravures sont réemployées par la suite, après la mort de Girardet en 1871, dans un volume de la B.J.C. intitulé la France catholique en Égypte, de Victor Guérin (1887). La pratique du réemploi est courante dans le cadre de l'édition industrielle, donc chez Mame. Par ailleurs, on remarquera qu'une image réalisée pour un lectorat adulte peut être réutilisée pour les plus jeunes et inversement: il ne semble pas y avoir chez Mame, au XIX siècle et pour cette collection en particulier ${ }^{24}$, de traitement spécifique de l'illustration pour les enfants: les images qu'Alfred Mame commande à ses artistes pour la B.J.C. conviennent tout autant aux adultes qu'aux plus jeunes.

Paradoxalement, c'est donc à partir de vues, de croquis et de souvenirs datant du début des années 1840 que le lecteur peut découvrir les paysages égyptiens dans les années 1880 . On trouve même une bataille d'Héliopolis, sujet auquel le peintre avait travaillé à la fin des années 1830. Cette distance chronologique oblige parfois à quelques mises au point. Ainsi de l'obélisque dans une vue d'Alexandrie publiée telle quelle en 1860, mais dont on précise en 1887 en légende que « l'obélisque encore debout, marqué ici près d'un autre renversé, a depuis quelques années, de même que celui-ci, été transporté hors de l'Égypte ». Girardet continue à décliner le genre du paysage selon le mode du pittoresque: on trouve dans la France catholique en Égypte une vue de la mosquée du sultan Hassan, au Caire où la solennité du monument est amoindrie par une scène de marché en premier plan, et beaucoup d'autres exemples où la composition tient davantage lieu de la règle d'atelier que d'une fidélité au paysage d'origine.

\section{Le voyage pittoresque, entre histoire et géographie}

\footnotetext{
${ }^{23}$ Sur les thèmes montagnard et suisse dans les collections des éditions catholiques pour la jeunesse, voir MARCoIN, Francis, Librairie de jeunesse et littérature industrielle au XIXe siècle. Paris : Honoré Champion, 2006, p. 219-224.

${ }^{24}$ Albert Uriet, au début du Xx ${ }^{\mathrm{e}}$ siècle, illustrateur et directeur artistique chez Mame, adoptera - et fera adopter - au contraire un style plus naïf et « plat », supposé plus adapté à l'œil enfantin, avec des lignes très fermes et de grands aplats de couleurs sans nuances, selon une manière inaugurée en Grande-Bretagne dans les années 1860 par Walter Crane et popularisée en France par Boutet de Monvel. On pense notamment à l'album les Chevaliers de la table ronde illustré par Uriet en 1948, ou encore aux ouvrages illustrés par Marie-Madeleine Franc-Nohain au début des années 1930.
} 
Le genre éditorial du "voyage pittoresque », très à la mode durant la période romantique, réalise une synthèse entre l'histoire et le paysage. Son origine se trouve à la fois dans les guides touristiques, qui apparaissent au XVIII ${ }^{\mathrm{e}}$ siècle, et dans les albums d'estampes archéologiques qui alimentent la passion pour l'histoire des artistes et des hommes de lettres depuis la Révolution. Le Musée des monuments français d'Alexandre Lenoir trouve sa descendance dans le monde du livre et de l'image imprimée avec la gigantesque entreprise des Voyages pittoresques et romantiques dans l'ancienne France de Charles Nodier, Isidore Taylor et Alphonse de Cailleux, illustré de plus de 3000 lithographies dans 23 volumes publiés entre 1820 et $1881^{25}$.

Le genre du voyage pittoresque s'éteint après $1855^{26}$. C'est pourtant en 1855 qu'est publié le chef-d'œuvre bibliophilique de Mame, la Touraine, écrit par un groupe d'érudits locaux sous la houlette de l'abbé Bourassé, et exclusivement illustré par deux artistes paysagistes, François-Louis Français et Karl Girardet. Les deux hommes obtiendront d'ailleurs chacun une médaille d'honneur pour cet ouvrage à l'Exposition universelle de 1855. Ceci tendrait à montrer que la maison Mame, dans ce genre de productions en particulier, est en retard par rapport à la tendance nationale, et continue de produire des voyages pittoresques illustrés de gravures après son heure de gloire, les années 1820 et $1830^{27}$. Un autre exemple de voyage dans l'espace et dans le temps, à travers la description graphique et littéraire de ce que Pierre Nora appellera bien plus tard des " lieux de mémoires », est plus discret et surtout beaucoup plus tardif : c'est celui du Rhin allemand d'Hippolyte Durand, qui paraît, toujours avec des illustrations de Girardet, en 1865. Les voyages pittoresques n'en portent plus le nom comme à l'époque romantique, mais continuent bien avant dans le siècle. Dans la même veine "étrangère », on pourra mentionner la Suisse pittoresque de Paul Fribourg publié en 1880, qui contient 24 gravures sur bois gravées d'après Girardet. Cette dernière publication ressemble beaucoup à un hommage posthume à l'illustrateur, à travers un livre qui célèbre la géographie et l'histoire de sa patrie d'origine, dix ans après sa mort ${ }^{28}$.

On sort avec ces trois derniers ouvrages du livre pour enfants, mais les volumes archéologiques que publie Mame dans le cadre de la B.J.C. au début des années 1840 sont à situer dans la même lignée, comme l'Archéologie chrétienne de Bourassé, publié dans la collection depuis 1841 avec des vues de cathédrales

\footnotetext{
25 AdHEMAR, Jean, la France romantique, les lithographies de paysage au xixe siècle. Paris : Somogy, Fondation Taylor, 1997 (1937) ; FouCART, Bruno (dir.), Adrien Dauzats et les Voyages pittoresques et romantiques dans l'ancienne France du baron Taylor. Paris : Fondation Taylor, 1990 ; Voyages pittoresques : Normandie 1820-2009; cat. expo. Rouen, Le Havre, Caen. Milan : Silvana editoriale, 2009.

${ }^{26}$ Voir la thèse de Caroline Becker-Jeanjean, les Récits illustrés de voyages pittoresques publiés en France entre 1770 et 1855, dir. Jean-Michel Leniaud, à l'École des Chartes, Paris, en 1999.

${ }^{27} \mathrm{La}$ Touraine ne fait toutefois ici que lancer le mouvement des monographies régionales et départementales d'initiative locale, qui prennent le relais, dans la seconde moitié du siècle, des ouvrages d'initiative parisienne de la période romantique.

${ }^{28}$ Girardet mourant en 1871, les compositions sont donc soit des réemplois, soit des gravures posthumes exécutées à partir de ses dessins. La mention du nom de l'illustrateur en page de titre, très rare chez Mame en dehors des publications de luxe du type de la Touraine, tend à confirmer l'hypothèse d'un hommage posthume.
} 
gravées sur bois par Charles Thompson ${ }^{29}$, et dont l'une des gravures (Cathédrale de Tours, non signée) sera remplacée en 1862 (6e édition) par une vue de la cathédrale de Reims par Girardet, toujours gravée par Thompson ${ }^{30}$; ou bien encore Rome et Lorette de Louis Veuillot (3e édition en 1845), qui est derechef illustré par Girardet, le Girardet voyageur en Italie cette fois-ci. On retrouve encore le nom du peintre suisse attaché à des titres comme les Récits et souvenirs d'un voyage en Orient de Baptistin Poujoulat ( $2^{\mathrm{e}}$ édition en 1850), les Tableaux de la Grèce ancienne et moderne de Jules Lacroix de Marlès (7e édition en 1862), les Pélerinages de Suisse de Louis Veuillot (13e édition en 1869), etc.

Le caractère proprement chrétien de ces publications ne nous semble pas ressortir dans les images, et doit, nous semble-t-il, être mis en évidence essentiellement dans le texte. Un exemple paradigmatique, de ce point de vue, se situe dans les publications de l'abbé Bourassé, professeur au petit séminaire et membre honoraire de la Société archéologique de Touraine ${ }^{31}$, qui s'efforce de faire du patrimoine religieux l'acmé du patrimoine national. De ce point de vue chrétien, les voyages pittoresques peuvent également être mis en relation avec l'Itinéraire de Paris à Jérusalem de Chateaubriand, que Mame publie encore en 1876 avec deux hors-texte signés par Girardet (sur trois planches en tout), et qui opère un lien à la fois géographique et symbolique entre l'histoire de la France et celle du christianisme. Ce dernier ouvrage ne fait encore une fois pas partie d'une collection spécialement dédiée à la jeunesse, mais l'exemplaire conservé à la bibliothèque municipale de Tours contient encore un tampon d'école ${ }^{32}$ qui montre à quel point ce genre d'ouvrages traversait les classes d'âge.

\section{Conclusion}

Entre peinture d'histoire, peinture de genre et paysage, Girardet s'est taillé dans les années 1830 une réputation qui séduit la maison Mame quand elle envisage, au début des années 1840, de développer l'illustration de ses ouvrages pour la jeunesse. L'artiste suisse signe avec Français l'un des quatre chefsd'œuvre du livre illustré que produit Alfred Mame durant sa carrière d'éditeurimprimeur - le Missel de Louis Hallez, la Bible de Gustave Doré, la Chanson de Roland de François Chifflart, et la Touraine. Si le principal atout de Girardet semble être sa polyvalence, des Fables de La Fontaine aux paysages d'Égypte en passant par les monuments archéologiques nationaux, les illustrations au goût naïf des contes de Schmid et les scènes d'histoire de France, il reste que sa

\footnotetext{
${ }^{29}$ Graveur d'origine anglaise, Charles Thompson introduit en France, dans les années 1820, la technique anglaise du bois de bout qui révolutionnera la mise en page du livre romantique. Il signe ici ses dernières gravures.

${ }^{30}$ Cette gravure est en 1862 un réemploi de celle publiée en 1843 dans les Cathédrales de France, toujours de Bourassé, ouvrage qui ne fait manifestement pas partie d'une collection pour la jeunesse. La première édition de l'Archéologie chrétienne, en 1841, ne comporte aucun hors-texte mais des vignettes - non signées - à fonction strictement documentaire (coupes, schémas et plans architecturaux). Les hors-textes des éditions ultérieures sont des réemplois des gravures de Cathédrales de France de 1843, dont Rémi Blachon (la Gravure sur bois au XIXe siècle, l'âge du bois debout. Paris : Éditions de l'Amateur, 2001, p. 62) semble estimer que la totalité est attribuable à Girardet (deux hors-texte sur les huit sont signés).

31 Société dans les membres honoraires de laquelle on retrouve le nom du baron Taylor...

32 « Ecole municipale de garçons, groupe Mirabeau, ville de Tours », C5922 à la BM de Tours.
} 
« spécialité », l'un de ses principaux apports à la richesse de l'illustration des livres de Mame, est ce mélange d'histoire anecdotique et de paysage pittoresque qui caractérise le genre du voyage pittoresque. Peu présents du point de vue quantitatif, les voyages pittoresques forment néanmoins l'horizon de nombreuses publications illustrées de l'éditeur entre le début des années 1840 et la fin des années 1860, dont le monument de la Touraine, en 1855, est la partie la plus visible. Derrière telle illustration de l'Histoire de Louis XIV d'Amédée Gabourd (1846, Girardet), on peut voir l'anecdote tirée de l'histoire de France qui fait le ravissement des amateurs d'histoire nationale. Derrière la vignette de page de titre de l'Histoire de Marie Stuart, reine d'Écosse de de Marlès (1843, non signée), on devine un beau paysage pittoresque dans la lignée sinon de Bonington, du moins de Fragonard.

Davantage qu'un genre phare de l'éditeur, le voyage pittoresque constitue donc, dans le cadre pédagogique des livres de prix qui constitue la spécialité de la production illustrée de Mame à partir des années 1840, un modèle esthétique où se croisent l'anecdotique et le pittoresque. Entre ces deux pôles, un point commun : la volonté de retenir le regard par un sujet distrayant, ni trop violent ni trop classique, qui est caractéristique, selon Rosenthal ${ }^{33}$, de l'art du « juste milieu » sous la monarchie de Juillet.

$\mathrm{Au}$ croisement de l'histoire et de la géographie, un voyage pittoresque pour enfants comme le Voyage en France de Mme Amable Tastu constitue sur de nombreux points un épisode intermédiaire entre les Voyages pittoresques et romantiques dans l'ancienne France du baron Taylor et le Tour de la France par deux enfants de G. Bruno ${ }^{34}$. Comment passer de l'entreprise archéologicotouristique des Voyages pittoresques de Taylor au livre scolaire et patriotique de Bruno ? En passant par un genre de livres qui mêle l'engouement archéologique et l'esthétique du pittoresque, la passion pour l'histoire nationale et le souci d'instruction de la jeunesse.

\footnotetext{
33 Rosenthal, Léon, Du romantisme au réalisme, essai sur l'évolution de la peinture en France de 1830 à 1848. Paris : H. Laurens, 1914. Voir également sur ce concept RoSEN, Charles, ZERNER, Henri, «Le juste milieu et Thomas Couture », in Romantisme et réalisme, mythes de l'art du XIXe siècle, traduit de l'américain par Odile Demange. Paris : Albin Michel, 1986 (1984). On remarquera que Rosenthal fait du maître de Girardet, Léon Cogniet, l'un des principaux représentants, avec Delaroche et Vernet, de cette peinture qui privilégie l'intelligibilité immédiate, le sujet et l'anecdotique.

34 Patrick Cabanel (le Tour de la nation par des enfants, romans scolaires et espaces nationaux (XIXe-XXe siècles). Paris : Belin, 2007) a d'ailleurs bien mis en évidence l'aspect précurseur, entre autres ouvrages, de ce livre de Mme Tastu par rapport au best-seller de G. Bruno, mais il ne fait jamais référence au genre éditorial du voyage pittoresque, sa perspective étant plus celle de l'histoire de l'éducation que de l'histoire du livre ou de l'art.
} 